\title{
Impacto de choques exógenos petroleros sobre algunos indicadores macroeconómicos en el Ecuador
}

\author{
Impact of exogenous oil shocks on some \\ macroeconomic indicators in Ecuador
}

Campuzano Vásquez, John Universidad Técnica de Machala

(Machala - Ecuador) jcampuzano@utmachala.edu.ec

Salcedo-Muñoz, Virgilio Universidad Técnica de Machala (Machala - Ecuador) vsalcedo@utmachala.edu.ec

Bejarano Copo, Holger Universidad Técnica de Machala

(Machala - Ecuador) hbejarano@utmachala.edu.ec

Molero Oliva, Leobaldo Universidad del Zulia (Mracaibo - Venezuela) Molero Oliva, Leobaldo

Núñez Guale, Linda Universidad Península de Santa Elena (Santa Elena - Ecuador) lmolerooliva@gmail.com

Revista Cumbres Vol.5 №2

Versión impresa ISSN 1390-9541

Versión electrónica ISSN 1390-3365

http://investigacion.utmachala.edu.ec/revistas/index.php/Cumbres 


\title{
RESUMEN
}

El Ecuador es una economía pequeña y abierta, sin apenas influencia en las condiciones de los mercados internacionales y, por lo tanto, expuesta a choques exógenos, como un aumento de la tasa de interés internacional o un alza en el precio del petróleo. Así mismo, por su condición de economía dolarizada, la responsabilidad de estabilización de los ciclos y de la promoción del crecimiento recae en la política fiscal. El objetivo de esta investigación es estimar el impacto de los choques exógenos petroleros sobre algunos indicadores macroeconómicos de la economía ecuatoriana, en el período 2007-2017 con datos de frecuencia mensual. La metodología del trabajo es explicativa y emplea la técnica estándar de Vectores Autorregresivos (VAR) para analizar las funciones impulso-respuesta que resultan de la estimación, utilizando un esquema de identificación a través la descomposición de Cholesky. Los hallazgos empíricos revelan un impacto del mismo signo que va del precio del petróleo hacia el indicador que recoge la actividad económica, lo que significa que ante choques exógenos inesperados en el mercado petrolero la respuesta del producto será del mismo signo, pero dicho choque no influye en el nivel de precios al consumidor directamente, sino que lo hace a través de la demanda agregada.

Palabras clave: Choques exógenos petroleros, indicadores macroeconómicos, sector real, VAR.

\begin{abstract}
Ecuador is a small and open economy, with hardly any influence on the conditions of international markets, and therefore exposed to exogenous shocks, such as an increase in the international interest rate or an increase in the oil price. Likewise, due to its status as a dollarized economy, the responsibility for the stabilization of cycles and the promotion of growth lies with fiscal policy. The objective of this research is to estimate the impact of exogenous oil shocks on some macroeconomic indicators of the Ecuadorian economy, in the period 2007-2017 with monthly frequency data. The methodology of the work is explanatory and uses the standard technique of Autoregressive Vectors (VAR) to analyze the impulse-response functions that result from the estimation, using an identification scheme through the decomposition of Cholesky. The empirical findings reveal an impact of the same sign that goes from the oil price to the indicator that reflects the economic activity, which means that in the face of unexpected exogenous shocks in the oil market the response of the output will be of the same sign, but this shock does not influence at the level of consumer prices directly, but it does so through aggregate demand.
\end{abstract}

Keywords: Exogenous oil shocks, macroeconomic indicators, real sector, VAR.

\section{Cumbres}




\section{INTRODUCCIÓN}

El estudio de las posibles causas de las fluctuaciones económicas ha sido la mayor preocupación en macroeconomía durante los años recientes (Bjørnland, 2000). La actividad económica real, el empleo, el nivel de precios, la inflación, el tipo de cambio, el saldo presupuestario gubernamental, el equilibrio externo y las reservas internacionales, son algunas de las variables macroeconómicas afectadas por choques de naturaleza doméstica (reales o nominales) o por choques exógenos a la economía como, por ejemplo, avances tecnológicos o cambios en los precios de las materias primas.

En ese sentido, para Quero-Virla (2016:25) el petróleo es un componente clave en la economía global, y recibe una importante consideración porque se presume juega un importante rol para explicar a otras variables macroeconómicas (Cunado y Pérez, 2005). En particular, las fluctuaciones económicas originadas por cambios en el precio del petróleo llaman la atención desde que la crisis en el precio del petróleo en el año 1973 repercutió fuertemente en los países avanzados (Sachs, 1981; Iwayemi y Fowowe, 2011), pero al mismo tiempo aumentó de forma más que considerable el flujo de ingresos entre los países proveedores de esta materia prima.

Luego de la recesión de los años setenta, surge un conjunto significativo de literatura que estudia el efecto de los choques en los precios del petróleo sobre las variables macroeconómicas. Al respecto, Bjørnland lo sintetiza del siguiente modo: "the instability of the world economy in the aftermath of the oil price shocks in the 1970's brought a renewed interest in the study of business cycles" (Bjørnland, 2000).

Para los países importadores netos de petróleo, la elevación de los precios del petróleo promueve choques sobre la economía, incrementando el nivel de precios internos (canal de costos), depreciando la moneda local (canal del tipo de cambio), disminuyendo el producto (Malik, Ajmal y Zahid, 2017; Doğrul y Soytas, 2010), la demanda agregada (Cunado y Pérez, 2005) y, a mediano y largo plazo, reconfigurando su estructura productiva a través de las decisiones intertemporales de consumo, ahorro e inversión.

Del mismo modo, los países exportadores netos de petróleo se benefician de forma apreciable del aumento en el precio del petróleo (choques positivos externos), en primer lugar, a través del aumento en los ingresos fiscales de origen petrolero, y de ahí vía mayor gasto público que expande la demanda agregada.

No obstante, para los países exportadores netos de petróleo con arreglos fiscales que contemplan altos niveles de gasto por concepto de subsidios de energías, los aumentos en el precio del petróleo conllevan a una elevación del costo de oportunidad de emplear dichos recursos en otros bienes públicos (salud, educación, infraestructura) de no existir tales subsidios, al tiempo que constituye una fuerte restricción en períodos de caída en el precio del petróleo en los mercados mundiales, por la consabida y aceptada presunción de la rigidez a la baja que tiende a exhibir el gasto público ${ }^{1}$.

Como todo país exportador de petróleo, el Ecuador se beneficia de los altos precios internacionales de las energías. Sin embargo, también ha suce- 
dido que, según autores como Jurado, Bejarano, Salcedo y Sánchez (2017): “el mercado energético a nivel mundial ha presentado cambios repentinos en el precio internacional del barril de petróleo, generando de manera imprevista graves consecuencias económicas y sociales" (Jurado et al., 2017:34). De hecho, en años recientes a la par de la caída en el precio internacional del petróleo, el Ecuador desaceleró su ritmo de crecimiento, y más bien entró en recesión, a juzgar por los resultados del PIB real.

En un marco general, Palma (2011) llega a la conclusión de que las economías rentistas, al depender de actividades económicas cambiantes y volátiles sujetas a realidades externas fuera del control de las autoridades nacionales, se transforman en economías vulnerables y cambiantes, y es común en estas economías que coincidan episodios de cambios significativos en el precio del petróleo con una mayor volatilidad de la actividad económica y cambios apreciables en los precios internos.

Desde el plano empírico la cuestión del impacto que causa el precio del petróleo en los indicadores macroeconómicos ha sido abordada, entre otros, por Paladines y Paladines (2017) y Jurado et al. (2017), en el caso del Ecuador, por Quero-Virla (2016) en Colombia, por Foudeh (2017) para Arabia Saudí, Lee y Ni (2002) para economías emergentes, Zakaria y Shamsuddin (2017) en Malasia, y Alonso y Martínez (2017) para la Alianza del Pacífico, Cunado y Pérez (2005) para seis países de Asia, Jiménez y Sánchez (2005) para algunos países industrializados de la OCDE, Brown y Yücel (2002), y Abeysinghe (2001), entre otros.

En este papel de trabajo se emplean los datos disponibles para estimar el impacto de los choques exógenos de los precios del petróleo sobre algunos indicadores macroeconómicos del Ecuador, en un período histórico concreto de 96 meses que van desde el año 2010 (2010:1) hasta el año 2017 (2017:12). El período elegido está definido por fuerte choques positivos y negativos del precio del petróleo, y sus efectos, según nuestra hipótesis de estudio, se vislumbraron en el desempeño macroeconómico. En general, Brown y Yücel (2002) justifican que un cuerpo considerable de investigaciones económicas sugiere que las fluctuaciones del precio del petróleo han tenido una considerable influencia en la actividad económica nacional de los países desde hace tiempo.

Para la estrategia econométrica la investigación se basa en la metodología de los Vectores Autorregresivos (VAR), puesto que de los mismos se derivan las funciones impulso-respuesta a través de las cuales asumiremos el análisis de la relación entre precio del petróleo y dos indicadores de la actividad económica real y de los precios en un horizonte de diez períodos.

Para evitar las consecuencias que se derivan de los choques provenientes del sector externo de la economía, principalmente la variabilidad de los

${ }^{1}$ Aún más entre las economías emergentes que tienen una alta dependencia de los ingresos fiscales de origen petrolero y, en ausencia de fondos intergeneracionales, donde existe indicio de que la mayor parte de los países exportadores contemplan un alza del gasto público durante los períodos de incremento en el precio del petróleo, esto es, la política fiscal adopta una postura pro cíclica, aún más cuando el país es una democracia más o menos estable o el país tiene fuertes presiones por redistribuir la riqueza debido a la alta desigualdad. 
precios del petróleo, economías como la del Ecuador, con una alta dotación de recursos naturales, deben fijar entre sus objetivos a mediano plazo la diversificación económica.

\section{MATERIALES Y MÉTODOS}

Tres variables son consideradas por este estudio. El tipo de datos usados son series de tiempo y fueron recolectados de fuentes secundarias para el período 2010-2017 con frecuencia mensual. La data para la variable precios del petróleo (de aquí en adelante wti) es el marcador West Texas International (WTI), el producto real (de aquí en adelante iaecsa) es recogido por el Índice de Actividad Económica Coyuntural (2007=100) del Banco Central del Ecuador desestacionalizado previamente para eliminar el efecto estacional, y el nivel de precios (en adelante ipcsa) es el Índice de Precios al Consumidor, nacional general desestacionalizado $(2014=100)$ estimado por el Banco Central del Ecuador. Todas las variables se transformaron en una escala logarítmica. Las dos últimas recogen los indicadores macroeconómicos en estudio.

La figura 1 muestra el comportamiento temporal de las series, tanto en niveles (escala logarítmica) como en diferencias (una aproximación a su variación relativa entre períodos). Todas las series, en niveles, reflejan un comportamiento con tendencia común, pero sus primeras diferencias si muestran un comportamiento aleatorio o estacionario alrededor de su media. Esta inspección preliminar por supuesto debe validarse de una forma más robusta con las pruebas disponibles a tal fin.

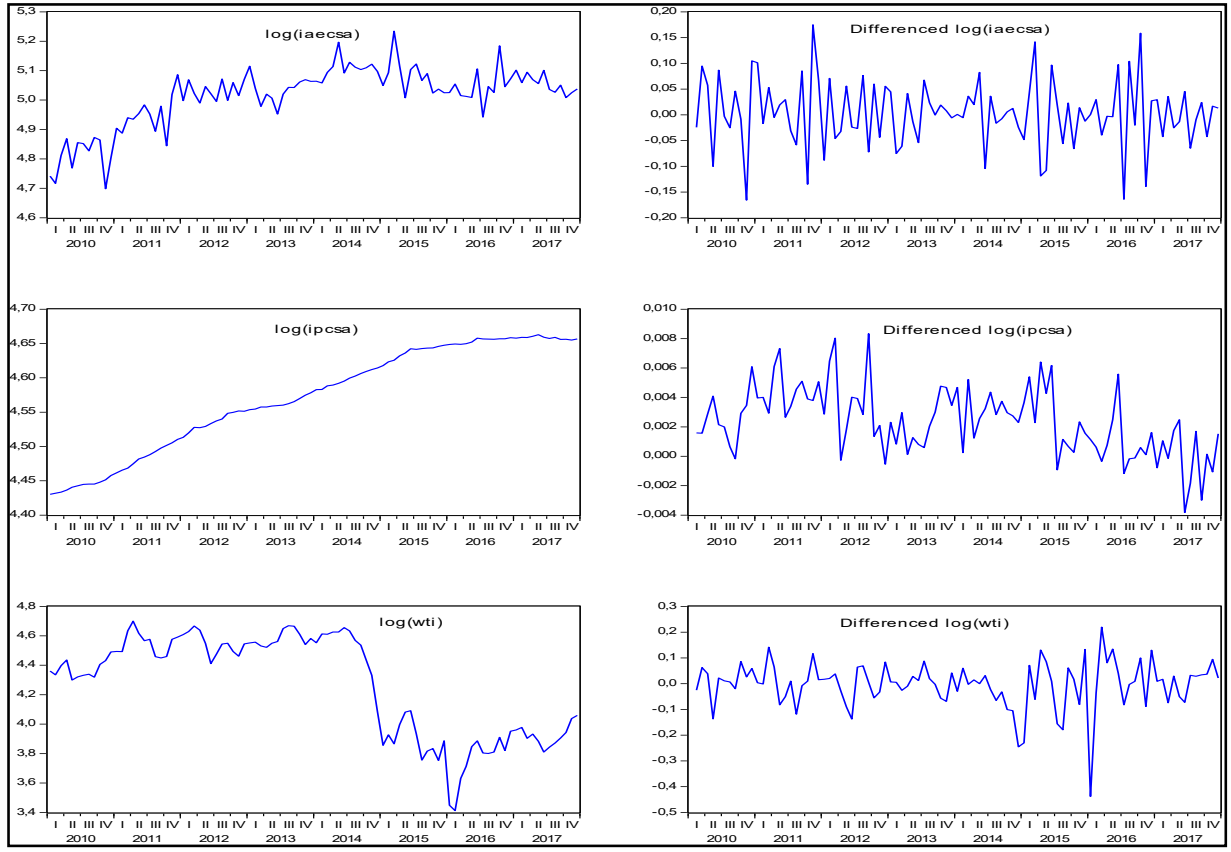

Fig. 1. Comportamiento del precio del petróleo, principales marcadores (2010:1-2017:12). 
La estimación del impacto de los precios del petróleo en los dos indicadores macroeconómicos de referencia del Ecuador sigue la tradición de los Vectores Autorregresivos (VAR). Un proceso VAR ha sido frecuentemente una elección popular para la descripción de datos macroeconómicos de series de tiempo, principalmente por su flexibilidad, facilidad de estimación y porque usualmente brinda una buena bondad de ajuste para los datos macroeconómicos (Juselius, 2006:14; Quero-Virla, 2016).

Las ecuaciones que especifican a un modelo VAR describen un sistema en el cual cada variable es una función de sus propios rezagos, de los rezagos de las otras variables en el sistema y los errores como variables exógenas. Un VAR es simplemente un proceso autorregresivo para un vector de variables.

Si se define $W_{t}=\left[\begin{array}{l}x_{t} \\ y_{t}\end{array}\right]$, una matriz $A_{2 \times 2}$ y $\epsilon_{t}=\left[\begin{array}{l}\epsilon_{1 t} \\ \epsilon_{2 t}\end{array}\right]$, entonces un VAR puede ser escrito como:

$$
\begin{aligned}
& W_{t}=A W_{t-1}+\epsilon_{t} \\
& \mathrm{O}, \text { también } \\
& x_{t}=a_{11} x_{t-1}+a_{12} y_{t-1}+\epsilon_{1 t} \\
& y_{t}=a_{21} x_{t-1}+a_{22} y_{t-1}+\epsilon_{2 t}
\end{aligned}
$$

Dicho en otras palabras, los modelos VAR brindan una forma de identificar las interacciones simultáneas entre las variables. Una condición previa que debe satisfacerse para el uso de VAR, es que las series deben contener el mismo orden de integración. Se evaluó el orden de integración de las variables mediante las pruebas Dickey-Fuller Aumentada (ADF) y Phillips-Perron (PP). Los resultados de estas pruebas se encuentran a continuación en la tabla 1.

Las pruebas indican que las variables son no estacionarias en niveles, salvo la variable iaecsa para la cual los resultados son ambiguos. La Prueba PP para esta serie, con constante y con tendencia, señala que es estacionaria en niveles al 5\%. Las primeras diferencias de las variables si fueron estacio-

\begin{tabular}{|c|c|c|c|c|}
\hline \multirow[b]{2}{*}{ Serie } & \multicolumn{2}{|c|}{ Pruebas de raíz unitaria ADF } & \multicolumn{2}{|c|}{ Pruebas de raíz unitaria PP } \\
\hline & Estadístico t & Valor Crítico $(\alpha=5 \%)$ & Estadístico t & Valor Crítico $(\alpha=5 \%)$ \\
\hline $\log$ (iaecsa) & $-2,442$ & $-3,459$ & $-4,786$ & $-3,458$ \\
\hline $\log (i p c s a)$ & 2,025 & $-3,458$ & 1,535 & $-3,458$ \\
\hline \multirow[t]{2}{*}{$\log (w t i)$} & $-2,021$ & $-3,458$ & $-2,021$ & $-3,458$ \\
\hline & \multicolumn{2}{|c|}{ Pruebas de raíz unitaria ADF } & \multicolumn{2}{|c|}{ Pruebas de raíz unitaria PP } \\
\hline Serie & Estadístico t & Valor Crítico $(\alpha=5 \%)$ & Estadístico t & Valor Crítico ( $\alpha=5 \%)$ \\
\hline dlog(iaecsa) & $-12,009$ & $-3,459$ & $-26,051$ & $-3,458$ \\
\hline dlog(ipcsa) & $-7,823$ & $-3,458$ & $-7,893$ & $-3,458$ \\
\hline$d \log (w t i)$ & $-8,713$ & $-3,458$ & $-8,687$ & $-3,458$ \\
\hline
\end{tabular}
narias, según los hallazgos con las pruebas señaladas. Dicho, en otros términos, son variables integradas de orden uno I (1). Estos resultados garantizan el mismo orden de integración entre las variables.

Tabla 1. Resultados Pruebas de Raíz Unitaria. 


\section{RESULTADOS}

Como puede verse en la figura 2, desde el año 2010 se presentaron fuertes cambios en el comportamiento de los precios del petróleo, según tres marcadores empleados (Crudo Oriente, NAPO y WTI); en efecto, se puede distinguir períodos de grandes aumentos como los de 2010-2011 (en promedio), de aumentos más moderados y aislados como los de mediados de 2012 hasta mediados de 2013, y, principalmente también, períodos de reducción de los precios, sobre todo el lapso que va desde 2014:6 (junio) hasta 2016:2 (febrero) que se hace claro en la figura referenciada, pues es el período que acusa la pendiente más descendente.

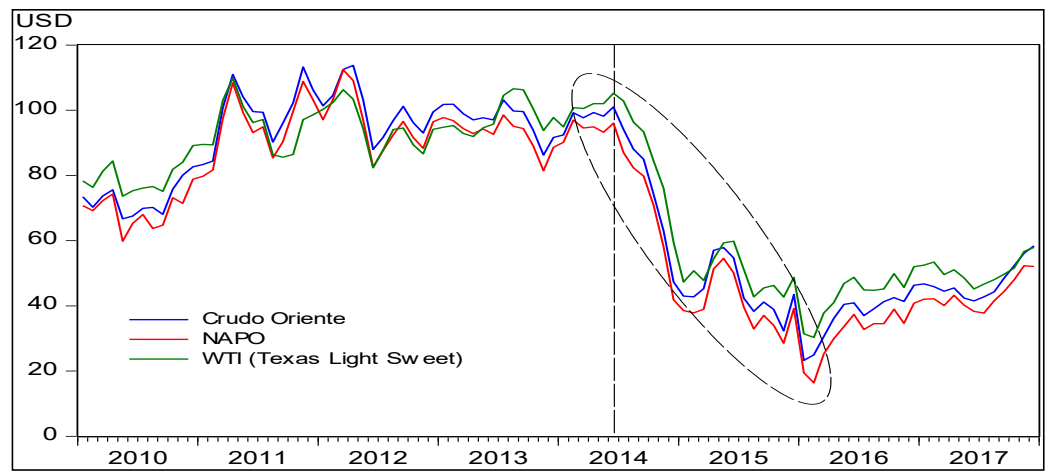

Fig. 2. Comportamiento del precio del petróleo, principales marcadores (2010:1-2017:12).

A un nivel de mayor desagregación, la figura 3 muestra el precio promedio de WTI, y de los otros dos (Crudo de Oriente y NAPO), en cada año. En efecto, en términos de promedio los años 2011, 2012 y 2013, disfrutaron de valores bastantes altos, sin embargo, el mercado demostró su volatilidad en cuanto el precio del petróleo comenzó a descender a mediados del año 2014, manifestándose en promedios muy bajos en los años 2015, 2016 y 2017. En ese sentido, se puede verificar como el precio promedio entre 2015 y 2017 se redujo para todos los marcadores a la mitad de los precios alcanzados entre 2011 y 2013. Otro síntoma de dicha volatilidad lo constituyen los valores para la desviación estándar, alrededor de la media, que adoptan cada una de estas series estadísticas en todo el período de análisis (véase la tabla 2).

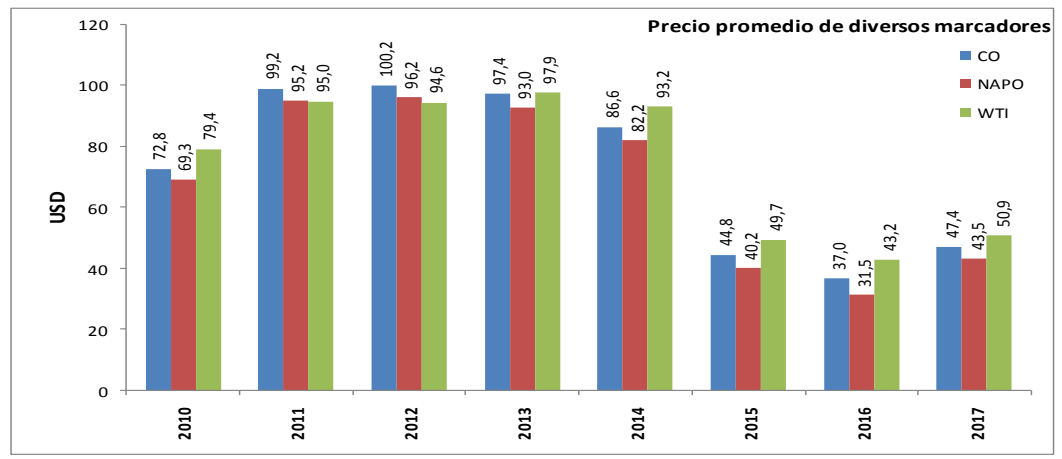

Fig. 3. Precio del petróleo, principales marcadores (Promedios por año). 
Tabla 2.Estadísticas descriptivas (2010:1-2017:12).

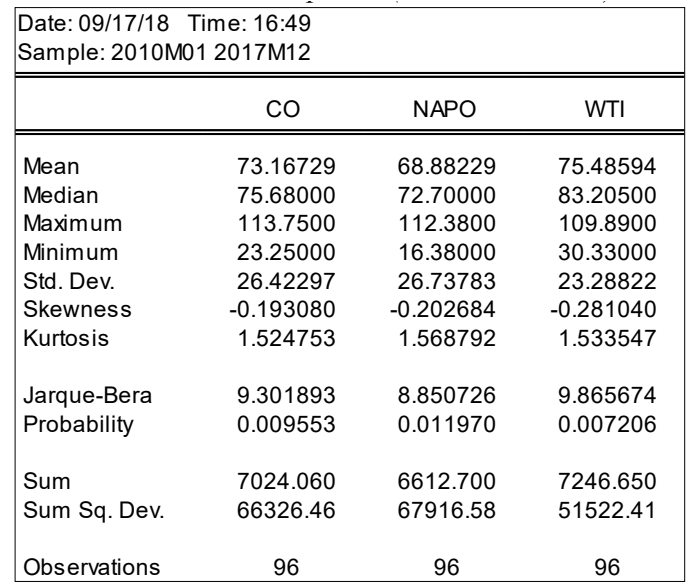

La desviación estándar para cada año se muestra también en la figura 4 . El análisis revela que el año 2014 fue, de la muestra, el más turbulento en cuanto al comportamiento de los precios. A principios de ese año, el precio del WTI se ubicó en el mismo nivel del mes de enero de 2013, y luego comenzó a crecer hasta el mes de junio, momento a partir del cual comienza un espectacular descenso que provocó que el nivel de del marcador llegara a poco menos de USD 60 en diciembre del 2014. Hasta entonces el precio del petróleo no había alcanzado, en el período de estudio, un nivel tan bajo. El descenso continuó en los años siguientes (2015 y 2016) pero a un ritmo más o menos estable, siendo el año 2016 quien presentó los precios mensuales más bajos. Estos niveles se recuperaron en el año 2017, aunque en valor absoluto siguen siendo más bajos que los niveles logrados antes del choque negativo en el mercado petrolero.

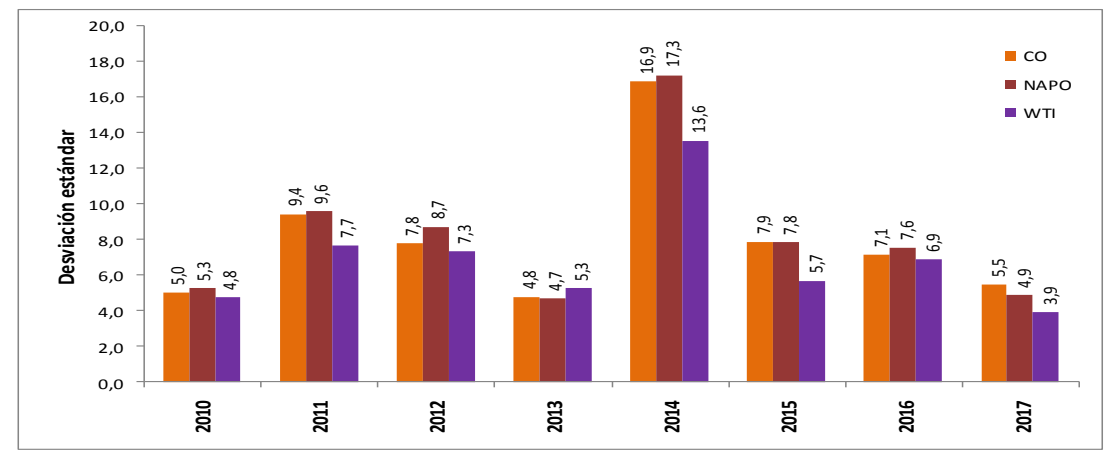

Fig. 4. Volatilidad del precio del petróleo (desviación estándar).

El modelo VAR estimado utiliza las series mensuales definidas en la sección anterior. La expectativa teórica es que, ante un choque en el nivel de precios del petróleo, dada la relevancia de este sector en el caso ecuatoriano, siga un incremento en el producto real y un aumento en el índice general de precios domésticos. 
Una vez analizado el orden de integración de las series, se procedió a efectuar la regresión con el programa Eviews 9. Los datos utilizados en el modelo VAR fueron: $\log (i a e c s a)$ : Logaritmo natural del Índice de Actividad Económica Coyuntural (2007=100) desestacionalizado; log(ipcsa): Logaritmo natural del Índice de Precios al Consumidor (IPC) nacional general desestacionalizado (2014=100); y, log(wti): Logaritmo natural del marcador WTI. La desestacionalización fue necesaria debido a que las series mostraban una fuerte estacionalidad, habida cuenta de sus frecuencias.

El modelo VAR lo componen tres (3) variables no estacionarias en niveles. Ante la imposibilidad de interpretar los coeficientes estimados del VAR, se procedió a la interpretación y discusión de las funciones impulso-respuesta (FIR), que miden los efectos de los diferentes choques sobre las variables de estudio. Las FIR, junto a la descomposición de varianza, son herramientas de los modelos VAR, provenientes de matrices especiales con las que se puede realizar el análisis respectivo. Las FIR para un total de 10 períodos se muestran en la Figura 5, para lo cual se utilizó el esquema de descomposición de Cholesky.

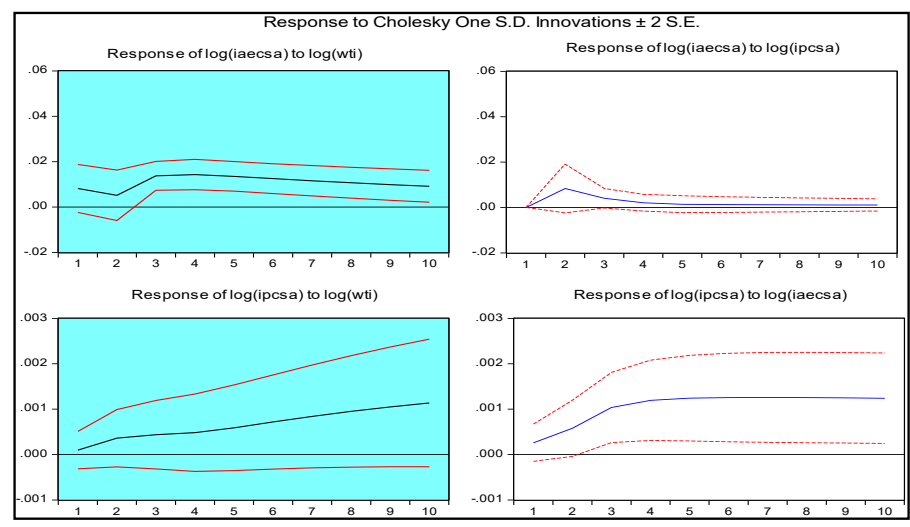

Fig. 5: Funciones Impulso-respuesta (FIR).

El VAR tiene el siguiente ordenamiento [ $(\log (w t i) \& \log (i a e c s a) \& \log (i p c s a))]$. En la selección del número de rezagos del VAR se consideraron los criterios de información Akaike, Schwarz y Hannan-Quinn. Estos criterios indican que uno (1) es el número de rezagos óptimos sugerido, sin embargo, se optó en principio por estimar un VAR con dos (2) rezagos para corregir problemas de autocorrelación.

En el lado derecho de la figura 5 se puede advertir que, según los resultados, un incremento en el precio del petróleo (choque exógeno positivo para el Ecuador como exportador neto) produce un incremento estadísticamente significativo en la actividad económica, a partir del tercer mes después del choque, mientras que en relación al impacto sobre el nivel de precios, genera un aumento en dicha variable pero no es, desde el punto de vista estadístico, significativo, durante el período de estudio.

En el caso del choque exógeno y su impacto sobre el producto real, como se ve un choque de dos (2) desviaciones estándar está asociado a un aumento 
promedio del PIB hasta un $2 \%$ hasta el décimo período, a partir del cual se reduce en los siguientes períodos. De este modo, el producto real agregado en Ecuador es procíclico respecto a los choques en los precios del petróleo, aunque con períodos de retraso, alcanzando su pico más alto en el tercer período luego del choque petrolero.

La evidencia respecto a la respuesta del nivel de precios ante estos mismos choques no es significativa estadísticamente, lo que podría significar de forma general que los precios responden a otro tipo de choques, podrían ser monetarios o de demanda interna. Esto se contrapone a la teoría, por cuanto se sabe que el nivel de precios responde, tanto a corto como a largo plazo, ante choques de oferta y de demanda.

En efecto, un choque de demanda, medido por el nivel de producto real de la economía, si tiene efectos sobre el nivel de precios, en el mismo sentido que se espera según los marcos teóricos de la inflación a corto plazo por presiones de demanda agregada.

La prueba gráfica de estabilidad estructural del VAR indica que todas las raíces características se localizan dentro del círculo unitario. El modelo estimado es estable. La prueba de criterios de elección del número óptimo de rezagos sugiere una selección de un solo rezago, como se había comentado anteriormente. La prueba de significancia de cada rezago confirma que sólo el rezago 1 es significativo dentro del VAR.

Una de las cuestiones claves que se pueden abordar con los VAR es la utilidad de algunas variables para pronosticar otras. La interrogante investigada es si un escalar y puede ayudar a pronosticar otro escalar x. Si no puede, entonces decimos que y no causa en el sentido de Granger a x. Se comprobó la causalidad con la prueba de Granger en bloque. Los resultados están contenidos en la tabla 7.

Tabla 7. Prueba de causalidad de Granger.

\begin{tabular}{|c|c|c|c|}
\hline \begin{tabular}{|l|} 
VAR Granger \\
Date: $09 / 21 / 18$ \\
Sample: 2010 \\
Included obse
\end{tabular} & $\begin{array}{l}\text { sality/Block } \\
\text { ime: } 08: 45 \\
12017 \mathrm{M} 12 \\
\text { tons: } 94\end{array}$ & & \\
\hline Dependent var & le: LOG(WT & & \\
\hline Excluded & Chi-sq & df & Prob. \\
\hline $\begin{array}{l}\text { LNIAECSA } \\
\text { LNIPCSA }\end{array}$ & $\begin{array}{l}1.889559 \\
2.887502\end{array}$ & $\begin{array}{l}2 \\
2\end{array}$ & $\begin{array}{l}0.3888 \\
0.2360\end{array}$ \\
\hline All & 7.229658 & 4 & 0.1242 \\
\hline Dependent var & le: LNIAECS & & \\
\hline Excluded & Chi-sq & df & Prob. \\
\hline $\begin{array}{l}\text { LOG(WTI) } \\
\text { LNIPCSA }\end{array}$ & $\begin{array}{l}16.30126 \\
24.08468\end{array}$ & $\begin{array}{l}2 \\
2\end{array}$ & $\begin{array}{l}0.0003 \\
0.0000\end{array}$ \\
\hline All & 24.09479 & 4 & 0.0001 \\
\hline Dependent var & le: LNIPCSA & & \\
\hline Excluded & Chi-sq & df & Prob. \\
\hline $\begin{array}{l}\text { LOG(WTI) } \\
\text { LNIAECSA }\end{array}$ & $\begin{array}{l}1.383178 \\
6.648434\end{array}$ & $\begin{array}{l}2 \\
2\end{array}$ & $\begin{array}{l}0.5008 \\
0.0360\end{array}$ \\
\hline All & 10.34677 & 4 & 0.0350 \\
\hline
\end{tabular}


La prueba sugiere lo que teóricamente se esperaba. La variable precio del petróleo es exógena, en el sentido de Granger, mientras que las variables macroeconómicas niveles de producto real y de precios no causan en el sentido de Granger a los precios internacionales del petróleo, variable que depende con toda seguridad de condiciones externas a la economía ecuatoriana, como por ejemplo eventos geopolíticos y económicos entre los países más relevantes a escala global en el sector y en la economía mundial.

La verificación del comportamiento ruido blanco se lleva a cabo mediante la estadística multivariada de Portmanteau, cuyos resultados confirman la ausencia de correlación serial. Igual conclusión se obtiene a partir de la prueba de los correlogramas y de la prueba de autocorrelación LM. Esto significa que el modelo está bien especificado. Los resultados de la prueba Jarque-Bera para normalidad de los residuos sugieren el rechazo de residuos multivariantes distribuidos como una normal; no obstante, no es relevante para el análisis de las funciones impulso-respuesta. Los resultados de la prueba de White para comprobar si los residuos son heterocesdásticos no rechazan la hipótesis de residuos homoscedásticos. La probabilidad del estadístico de prueba es mayor a 0,05.

Tabla 8. Descomposición de la varianza

\begin{tabular}{|c|c|c|c|c|}
\hline \multicolumn{5}{|c|}{ Variance Decomposition of LOG(WTI): } \\
\hline Perio... & S.E. & LOG(WTI) & LNIAECSA & LNIPCSA \\
\hline 1 & 0.088990 & 100.0000 & 0.000000 & 0.000000 \\
\hline 2 & 0.128617 & 99.15294 & 0.227325 & 0.619733 \\
\hline 3 & 0.153743 & 98.54532 & 0.210601 & 1.244082 \\
\hline 4 & 0.170367 & 98.21882 & 0.215681 & 1.565498 \\
\hline 5 & 0.182564 & 98.00603 & 0.209282 & 1.784683 \\
\hline 6 & 0.191872 & 97.82747 & 0.198283 & 1.974249 \\
\hline 7 & 0.199087 & 97.65917 & 0.187432 & 2.153401 \\
\hline 8 & 0.204723 & 97.49547 & 0.177933 & 2.326597 \\
\hline 9 & 0.209151 & 97.33375 & 0.170479 & 2.495775 \\
\hline 10 & 0.212645 & 97.17244 & 0.165539 & 2.662016 \\
\hline \multicolumn{5}{|c|}{ Variance Decomposition of LNIAECSA: } \\
\hline Perio... & S.E. & LOG(WTI) & LNIAECSA & LNIPCSA \\
\hline 1 & 0.051538 & 2.530585 & 97.46941 & 0.000000 \\
\hline 2 & 0.053186 & 3.324115 & 94.21081 & 2.465071 \\
\hline 3 & 0.055317 & 9.257491 & 87.91830 & 2.824214 \\
\hline 4 & 0.057279 & 14.89490 & 82.33937 & 2.765729 \\
\hline 5 & 0.058952 & 19.33531 & 77.99567 & 2.669019 \\
\hline 6 & 0.060337 & 22.76625 & 74.64096 & 2.592782 \\
\hline 7 & 0.061498 & 25.47694 & 71.98886 & 2.534203 \\
\hline 8 & 0.062474 & 27.63998 & 69.87021 & 2.489802 \\
\hline 9 & 0.063297 & 29.38032 & 68.16278 & 2.456904 \\
\hline 10 & 0.063991 & 30.79246 & 66.77430 & 2.433246 \\
\hline \multicolumn{5}{|c|}{ Variance Decomposition of LNIPCSA: } \\
\hline Perio... & S.E. & LOG(WTI) & LNIAECSA & LNIPCSA \\
\hline 1 & 0.001995 & 0.244589 & 1.690304 & 98.06511 \\
\hline 2 & 0.003053 & 1.496437 & 4.305394 & 94.19817 \\
\hline 3 & 0.003930 & 2.138400 & 9.531381 & 88.33022 \\
\hline 4 & 0.004700 & 2.541889 & 13.12343 & 84.33468 \\
\hline 5 & 0.005380 & 3.138462 & 15.33454 & 81.52700 \\
\hline 6 & 0.005991 & 3.964086 & 16.74776 & 79.28815 \\
\hline 7 & 0.006548 & 4.962951 & 17.70422 & 77.33283 \\
\hline 8 & 0.007062 & 6.076451 & 18.37102 & 75.55253 \\
\hline 9 & 0.007543 & 7.260227 & 18.84157 & 73.89820 \\
\hline 10 & 0.007995 & 8.480842 & 19.17416 & 72.34500 \\
\hline & 促 & VTI) L & & \\
\hline
\end{tabular}

Otro instrumento utilizado es la descomposición de la varianza donde se obtienen distintos componentes que permitirán aislar el porcentaje de variabilidad de cada variable que es explicado por la perturbación de cada ecuación, 
pudiéndose interpretar como la dependencia relativa que tiene cada variable sobre el resto. La descomposición de la varianza indica información sobre la cantidad en la que cada variable contribuye a explicar al resto de variables en el sistema. La tabla 8 brinda los resultados de la descomposición de la varianza. Cuando analizamos la tabla, se puede observar que la variabilidad del producto real puede ser explicada por los choques petroleros hasta casi un $31 \%$ al cabo de 10 períodos (meses). Los efectos de los choques petroleros comienzan a transmitirse desde el primer período, es decir, hay efectos contemporáneos en el producto real ante cambios en el precio del petróleo en el período estudiado.

En el caso del nivel de precios, los choques petroleros no son relevantes para explicar su comportamiento en los diez meses posteriores a la ocurrencia de una innovación; sin embargo, las variaciones en el nivel de producto real, como una expansión o choque de demanda agregada, si tienen efectos significativos y que perduran al cabo de unos cuantos períodos, al mismo tiempo que la variable parece ser explicada en una alta proporción por choques propios.

Tabla 9. Prueba de Cointegración de Johansen.

\begin{tabular}{|c|c|c|c|c|}
\hline \multicolumn{5}{|c|}{$\begin{array}{l}\text { Date: 09/21/18 Time: 10:44 } \\
\text { Sample (adjusted): } 2010 \mathrm{M} 04 \text { 2017M12 } \\
\text { Included observations: } 93 \text { after adjustments } \\
\text { Trend assumption: Quadratic deterministic trend } \\
\text { Series: LOG(WTI) LNIAECSA LNIPCSA } \\
\text { Lags interval (in first differences): } 1 \text { to } 2 \\
\\
\text { Unrestricted Cointegration Rank Test (Trace) }\end{array}$} \\
\hline $\begin{array}{c}\text { Hypothesized } \\
\text { No. of CE(s) }\end{array}$ & Eigenvalue & $\begin{array}{l}\text { Trace } \\
\text { Statistic }\end{array}$ & $\begin{array}{c}0.05 \\
\text { Critical Value }\end{array}$ & Prob.* ${ }^{* *}$ \\
\hline $\begin{array}{l}\text { None * } \\
\text { At most } 1 \\
\text { At most } 2\end{array}$ & $\begin{array}{l}0.322197 \\
0.057706 \\
6.09 E-06\end{array}$ & $\begin{array}{l}41.69590 \\
5.528336 \\
0.000566\end{array}$ & $\begin{array}{l}35.01090 \\
18.39771 \\
3.841466\end{array}$ & $\begin{array}{l}0.0084 \\
0.9031 \\
0.9828\end{array}$ \\
\hline \multicolumn{5}{|c|}{$\begin{array}{l}\text { Trace test indicates } 1 \text { cointegrating eqn(s) at the } 0.05 \text { level } \\
{ }^{*} \text { denotes rejection of the hypothesis at the } 0.05 \text { level } \\
{ }^{* *} \text { MacKinnon-Haug-Michelis (1999) p-values } \\
\text { Unrestricted Cointegration Rank Test (Maximum Eigenvalue) }\end{array}$} \\
\hline $\begin{array}{l}\text { Hypothesized } \\
\text { No. of } \mathrm{CE}(\mathrm{s})\end{array}$ & Eigenvalue & $\begin{array}{l}\text { Max-Eigen } \\
\text { Statistic }\end{array}$ & $\begin{array}{c}0.05 \\
\text { Critical Value }\end{array}$ & Prob.** \\
\hline $\begin{array}{l}\text { None * } \\
\text { At most } 1 \\
\text { At most } 2\end{array}$ & $\begin{array}{l}0.322197 \\
0.057706 \\
6.09 E-06\end{array}$ & $\begin{array}{l}36.16757 \\
5.527770 \\
0.000566\end{array}$ & $\begin{array}{l}24.25202 \\
17.14769 \\
3.841466\end{array}$ & $\begin{array}{l}0.0009 \\
0.8600 \\
0.9828\end{array}$ \\
\hline \multicolumn{5}{|c|}{$\begin{array}{l}\text { Max-eigenvalue test indicates } 1 \text { cointegrating eqn(s) at the } 0.05 \text { level } \\
{ }^{*} \text { denotes rejection of the hypothesis at the } 0.05 \text { level } \\
{ }^{* *} \text { MacKinnon-Haug-Michelis (1999) p-values }\end{array}$} \\
\hline
\end{tabular}

Probablemente, en el caso del Ecuador un choque positivo en el precio del petróleo expande el producto, pero no causa incrementos por sí mismo en el nivel de precios, habido cuenta que las energías gozan de ciertos subsidios en el mercado interno; pero, la expansión del ingreso como consecuencia del aumento en el producto, origina un aumento en la demanda interna por bienes y servicios finales, por esta vía termina influyendo o presionando el costo 
de vida en el Ecuador, al menos según lo que se deriva del período estudiado en esta investigación. Es decir, opera el canal de la demanda, pero no el canal de la oferta en los choques típicos en el precio del crudo sobre la economía ecuatoriana.

Finalmente, el resultado empírico de la estimación del VAR concluye con la prueba de cointegración de Johansen, habida cuenta que todas las series son integradas de orden uno. Cabe destacar que más allá del impacto que estudiamos del precio del petróleo sobre dos de las principales variables macroeconómicas, desde el punto de vista estadístico una de las virtudes que contienen los modelos VAR es que dejan abierta la posibilidad de combinar información de corto y largo plazo en los datos para explorar la propiedad de cointegración entre las variables. Quizás por ello los modelos VAR continúan recibiendo mucha atención de los econometristas y los economistas empíricos (Juselius, 2006). La Prueba de Cointegración de Johansen, dispuesta en la Tabla 9, indica la existencia de al menos una relación de cointegración a largo plazo entre las variables del estudio, significativa al $5 \%$.

\section{CONCLUSIONES}

Las razones por las cuales existen fluctuaciones económicas a corto plazo son relevantes tanto a nivel académico como desde el punto de vista de los decisores de políticas. Las fluctuaciones imprevistas o no anticipadas se traducen en costos en términos de pérdida de producto y empleo (costos reales) o por un alza en el nivel de precios que desvía la tasa inflación del valor previsto en el caso de una política de metas de inflación, por ejemplo.

Para las economías emergentes pequeñas la restricción es mayor, pues por sus características son susceptibles de captar choques de naturaleza exógena, sobre todo de los mercados internacionales de bienes y financieros. El Ecuador no escapa a esta realidad. Como país productor y exportador neto de petróleo, las exportaciones petroleras tienen un peso importante dentro de las exportaciones totales, de modo que las variaciones en el precio de esta materia prima abren un canal a través del cual puede fluctuar significativamente la demanda agregada y, por lo tanto, la actividad económica y el nivel de precios de la economía, indicadores macroeconómicos claves atados a dos de los principales objetivos de política económica.

Partiendo de estas consideraciones, y dada la experiencia empírica en este campo, se propuso en esta investigación estimar los choques exógenos en el precio del petróleo sobre dos indicadores macroeconómicos de la economía ecuatoriana: el producto real y el nivel de precios de la economía, en el período más reciente 2010-2017 con datos de frecuencia mensual, y a partir de la metodología econométrica de Vectores Autorregresivos (VAR).

Los resultados alcanzados no difieren del grado de consenso en la materia. Según ellos, un choque exógeno proveniente de las variaciones del precio del petróleo causa un impacto en la misma dirección y significativo estadísticamente sobre el nivel de producto real de la economía, representado por 
el indicador de la actividad económica coyuntural que publica la autoridad monetaria del Ecuador. Dicho impacto es prolongado y, en términos de magnitud, representativo.

La teoría en este caso sugiere que, para los países exportadores de crudo, un alza en el precio de este bien en los mercados mundiales, se traduce en una mejora en los términos de intercambio, en el valor de las exportaciones, y en promedio significa un alza a corto plazo en el ingreso de los agentes, expandiéndose la demanda agregada, dado el efecto del multiplicador. Además de ello la política fiscal, como regularidad empírica en los países exportadores de crudo, tiende a seguir el ciclo y amplia los efectos del alza en el precio del petróleo vía un mayor gasto público.

En el caso del nivel de precios, los hallazgos empíricos de este trabajo revelan efectos positivos, pero no significativos de los choques petroleros, aunque el incremento en el nivel de actividad económica si se traduce en presiones sobre el nivel de precios.

Como se sabe, en países con tipo de cambio flexible un incremento en el nivel de exportaciones, repentino dado el choque exógeno, se traduce en presiones sobre la moneda local, en concreto, la misma tiende a sufrir apreciaciones del tipo de cambio. En países con tipo de cambio fijo, cuyo extremo es la dolarización, dado el compromiso de la autoridad monetaria, el alza en el nivel de exportaciones y el superávit en cuenta corriente se traduce en presiones de demanda agregada sobre la oferta, por lo que puede ocurrir, incluso en ausencia de un patrón monetario propio, apreciaciones del tipo de cambio real por el aumento en la demanda de bienes no transables principalmente.

Se llama la atención sobre algunas vulnerabilidades que pudiese presentar la economía ecuatoriana, por la combinación de choques exógenos y características institucionales y estructurales que, en promedio, exhiben economías como la ecuatoriana.

\section{REFERENCIAS BIBLIOGRÁFICAS}

Abeysinghe, T. (2001). Estimation of direct and indirect impact of oil price on growth. Economics Letters, 73(2001), 147-153.

Alonso, J., \& Martínez, D. (2017). Impacto del precio del petróleo sobre el PIB de los países de la Alianza del Pacífico. Revista Finanzas y Política Económica, 9(2), 249-264.

Bjørnland, H. (2000). VAR models in macroeconomic research. Statistics Norway, Research Department, Documents 2000/14, October 2000.

Brown, S., \& Yücel, M. (2002). Energy prices and aggregate economic activity: an interpretative survey. The Quarterly Review of Economics and Finance, 42(2002), 193-208.

Cunado, J., \& Pérez, F. (2005). Oil prices, economic Activity and Inflation: evidence for some Asian countries. The Quarterly Review of Economics and Finance, 45(2005), 65-83.

Dickey, D., Jansen, D., \& Thornton, D. (1991). A primer on cointegration with an application to money and income.

Doğrul, H., \& Soytas, U. (2010). Relationship between oil prices, interest rate, and unemploy- 
ment: Evidence from an emerging market. Energy Economics, 32(6), 1523-1528.

Foudeh, M. (2017). The long run effects of oil prices on economic growth: the case of Saudi Arabia. International Journal of Energy Economics and Policy, 7(6), 171-192.

Iwayemi, A., \& Fowowe, B. (2011). Impact of oil price shocks on selected macroeconomic variables in Nigeria. Energy Policy, 39(2011), 603-612.

Jiménez, R., \& Sánchez, M. (2005). Oil price shocks and real GDP growth: empirical evidence for some OECD countries. Applied Economics, 37(2005), 201-228.

Jurado, C., Bejarano, H., Salcedo, V., \& Sánchez, M. (2017). La volatidad del precio del petróleo: sus efectos en la economía ecuatoriana en la última década. $3 C$ Empresa, 6(2), 33 - 45 .

Juselius, K. (2006). The cointegrated VAR model. Methodology and applications. Advanced texts in Econometrics. Editors M. Arellano, G. Imbens, G.E. Mizon, A. Pagan, y M. Watson. Oxford University Press.

Lee, K., \& Ni, S. (2002). On the dynamic effects of oil price shocks: A study using industry level data. Journal of Monetary Economics, 49(4), 823-852.

Malik, K., Ajmal, H., \& Zahid, M. (2017). Oil price shock and its impact on the macroeconomic variables of Pakistan: A Structural Vector Autoregressive approach. International Journal of Energy Economics and Policy, 7(5), 83-92.

Paladines, J. \& Paladines, A. (2017). Consequences of oil and food price shocks on the Ecuadorian economy. International Journal of Energy Economics and Policy, 7(3), 146-151.

Palma, P. (2011). Riesgos y consecuencias de las economías rentistas. El caso de Venezuela. Revista Problemas del Desarrollo, 165(42), 35-59.

Quero-Virla, L. (2016). Macroeconomic effects of oil price fluctuations in Colombia. Ecos de Economía: A Latin American Journal of Applied Economics, 20(43), 23-38.

Sachs, J. (1981). The current account and macroeconomic adjustment in the 1970s. Brooking Paper on Economic Activity, 1, 201-282.

Zakaria, Z., \& Shamsuddin, S. (2017). Causality relationship between crude oil variables and budget variables in Malaysia. International Journal of Energy Economics and Policy, $7(2), 132-138$. 